\title{
Lumped model for rotational modes in phononic crystals
}

\author{
Pai Peng, ${ }^{1}$ Jun Mei, ${ }^{2}$ and Ying $\mathrm{Wu}^{1, *}$ \\ ${ }^{1}$ Division of Mathematical and Computer Sciences and Engineering, King Abdullah University of Science and Technology (KAUST), \\ Thuwal 23955-6900, Saudi Arabia \\ ${ }^{2}$ Department of Physics, South China University of Technology, Guangzhou 510641, China
}

(Received 22 July 2012; published 16 October 2012)

\begin{abstract}
We present a lumped model for the rotational modes induced by the rotational motion of individual scatterers in two-dimensional phononic crystals comprised of square arrays of solid cylindrical scatterers in solid hosts. The model provides a physical interpretation of the origin of the rotational modes, reveals the important role played by the rotational motion in determining the band structure, and reproduces the dispersion relations in a certain range. The model increases the possibilities of manipulating wave propagation in phononic crystals. In particular, expressions derived from the model for eigenfrequencies at high symmetry points unambiguously predict the presence of a new type of Dirac-like cone at the Brillouin center, which is found to be the result of accidental degeneracy of the rotational and dipolar modes.
\end{abstract}

DOI: 10.1103/PhysRevB.86.134304

PACS number(s): 62.30.+d, 62.65.+k, 62.20.-x, 43.35.+d

\section{INTRODUCTION}

Phononic crystals, that is, structured materials with periodic modulations in their density and elastic coefficients, ${ }^{1-18}$ have been rapidly developed in recent years due to their applications in manipulating acoustic and elastic waves. Based on their constituents, phononic crystals can be categorized into at least two types. Prominent ones are acoustic crystals, which are solid/fluid inclusions in fluid hosts. ${ }^{1-8}$ Another common type is arrays of solid inclusions embedded in solid hosts. ${ }^{9-18}$ To differentiate this type from acoustic crystals, we call them elastic phononic crystals (EPCs). EPCs are more complex than their acoustic counterparts because of the shear deformation and induced shear-restoring force in solids, features that do not appear in ideal fluids. Here, ideal fluids refer to inviscous liquids or gases. ${ }^{19}$ The existence of shear deformation in an EPC is the underlying reason for many salient phenomena of this type of phononic crystal. One such phenomenon is the local rotational motion ${ }^{12,14,17}$ in an EPC, which does not exist in an acoustic crystal.

Although rotational motion has been extensively observed in EPCs, ${ }^{12,14,17}$ to the best of our knowledge, it has not yet been modeled. Without a model, it is difficult to accomplish the task of establishing the relationship between rotational modes and the microstructure of an EPC, which is crucial in utilizing the rotational modes to achieve intriguing wave propagation properties with potential applications. In the past it seems that research about EPCs was more focused on controlling the usual longitudinal and transverse modes ${ }^{10,11,13-15}$ in the crystal rather than on the physics of the rotational modes. Without a clear physical picture of the rotational motion, however, it is difficult to fully understand the wave propagation properties in an EPC.

In this paper we establish a model that describes the rotational modes in two-dimensional EPCs. We show that a simple lumped model captures the essence of the physics of the rotational modes, as well as offers a reasonably good estimation of the band structures of EPCs. Our model reveals the inherent link between the properties of the rotational modes and the microstructure of the EPC, and allows us to engineer an EPC that exhibits an unusual Dirac-like cone $e^{6,20,21}$ at the Brillouin center. The newly observed Dirac-like cone is obtained by exploiting the rotational motion to achieve accidental degeneracy of a rotational mode and dipolar modes.

\section{LUMPED MODEL}

The EPC considered in this work is a square array of cylindrical solid inclusions with radii $R$ embedded in another solid matrix. The lattice constant is $a$, which is also the length unit. The elastic wave propagation in such a system is characterized by the displacement $\mathbf{u}(\mathbf{r})$ of each point in the EPC. We focus on the wave propagation along the $\Gamma X$ direction and propose the lumped model shown in Fig. $1 . m_{1}$ and $m_{2}$ are the masses of the matrix and the scatterer, respectively, and the interaction forces between these masses are modeled by massless "springs" connecting them. ${ }^{22,23}$ In fact, these springs are "continuous" springs, but for simplicity and without loss of generality, we demonstrate in Fig. 1 by using two individual springs connected to the scatterer at two arbitrary points "A" and "B" on the boundary of the scatterer. To study the low frequency rotational modes, where the rotational vibration is more likely to occur in the scatterer about its axis, the mass $m_{2}$ is considered to be a finite-sized cylinder with radius $R$ and a moment of inertia $I_{2}$, rather than a point mass like $m_{1}$. The finite-size assumption was also used in the study of granular systems,${ }^{24,25}$ where the moment of inertia comes into play. A rotational mode naturally satisfies $\nabla \times \mathbf{u} \neq 0$, which means that the transverse wave is required to excite the rotational motion as $\nabla \times \mathbf{u}_{l}=0$ for longitudinal waves. In the low frequency limit it can be shown that along the $\Gamma X$ direction, only transverse waves are coupled with rotational motion. Therefore, we consider only transverse motion here as the translational movement. As a result, the displacements of the centers of masses are confined along the vertical direction and denoted by $u_{2 i-1}$ and $u_{2 i}$ for $m_{1}$ and $m_{2}$, respectively, where $i$ is the index of the unit cell.

The intermass force arises because of the relative distortion between the adjacent masses. There are two sources for the restoring force exerted on $m_{1}$ in the $i$ th unit cell. One is the translational distortion, that is, the relative 
(a)

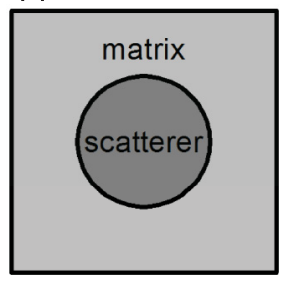

(b)

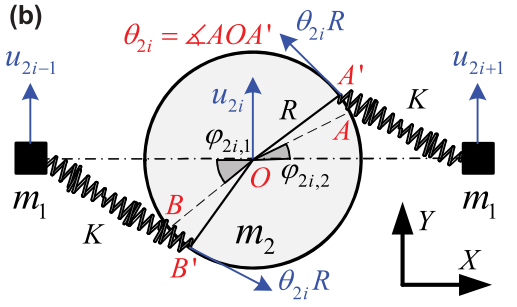

FIG. 1. (Color online) (a) The unit cell of the EPC. (b) A schematic of the lumped model. The solid black squares indicate the point-mass $m_{1}$ representing the matrix, and the circles indicate the finite-sized cylindrical scatterer with mass $m_{2} . u$ is the transverse displacement. A and B are arbitrarily chosen points on the boundary of the scatterer, whose equilibrium polar angles are $\varphi_{2 i, 1}\left(\varphi_{2 i, 2}\right)$. They rotate at an angle of $\theta_{2 i}$ about the axis of the cylinder from their equilibrium positions to points $A^{\prime}$ and $B^{\prime}$. The springs represent the restoring force between two adjacent masses.

displacement between the centers of the matrix and the scatterers nearby, which can be expressed as $\mathbf{F}_{t_{-} 2 i-1}=$ $-K\left[\left(u_{2 i-1}-u_{2 i}\right)+\left(u_{2 i-1}-u_{2(i-1)}\right)\right] \hat{y}$, where $K$ is the spring constant and is related to both the bulk and shear moduli of the matrix. ${ }^{23}$ The other source is introduced by the rotational movements of the two neighboring scatterers and takes the form $\mathbf{F}_{r_{-} 2 i-1}=\left[-\frac{K}{\pi} \int_{-\pi / 2}^{\pi / 2} \theta_{2 i} R \cos \varphi_{2 i, 1} d \varphi_{2 i, 1}+\right.$ $\left.\frac{K}{\pi} \int_{-\pi / 2}^{\pi / 2} \theta_{2(i-1)} R \cos \varphi_{2(i-1), 2} d \varphi_{2(i-1), 2}\right] \hat{y}$, where $\theta$ denotes the rotated angle of the scatterer from its equilibrium position and $\varphi_{2 i, 1}\left(\varphi_{2 i, 2}\right)$ is the polar angle before deformation. Similarly, the total restoring force exerted on $m_{2}$ can be written as $\mathbf{F}_{t-2 i}=$ $-K\left[\left(u_{2 i}-u_{2 i-1}\right)+\left(u_{2 i}-u_{2 i+1}\right)\right] \hat{y}$ and $\mathbf{F}_{r-2 i}=0$, which, respectively, comes from the relative distortion resulting from the translational and rotational movements of the scatterer. The rotation of the scatterer would not generate a net total force on itself, that is, $\mathbf{F}_{r_{-} 2 i}=0$, but it causes a torque on $m_{2}$ due to the finite size of $m_{2}$. Analogous to the resorting force, there are also two sources for the torque, which can be expressed as $\mathbf{M}_{t_{-} 2 i}=\left[\frac{K R}{\pi} \int_{-\pi / 2}^{\pi / 2}\left(u_{2 i}-u_{2 i-1}\right) \cos \varphi_{2 i, 1} d \varphi_{2 i, 1}-\right.$ $\left.\frac{K R}{\pi} \int_{-\pi / 2}^{\pi / 2}\left(u_{2 i}-u_{2 i+1}\right) \cos \varphi_{2 i, 2} d \varphi_{2 i, 2}\right] \hat{z}$ for the translational part, and $\mathbf{M}_{r_{-} 2 i}=-2 K\left(\theta_{2 i} R\right) R \hat{z}$ for the rotational part.

Newton's second law gives us the equations of motion for $m_{1}$ and $m_{2}$ :

$$
\begin{aligned}
m_{1} \ddot{\mathbf{u}}_{2 i-1} & =\mathbf{F}_{t \_2 i-1}+\mathbf{F}_{r_{-} 2 i-1}, \\
m_{2} \ddot{\mathbf{u}}_{2 i} & =\mathbf{F}_{t \_2 i}+\mathbf{F}_{r_{-} 2 i}, \\
I_{2} \ddot{\theta}_{2 i} & =\mathbf{M}_{t \_2 i}+\mathbf{M}_{r_{-2} 2 i} .
\end{aligned}
$$

Supposing time harmonic vibration and invoking Bloch theorem, Eq. (1) is transformed into the following secular equation:

$$
\begin{aligned}
& \operatorname{det}\left|\begin{array}{ccc}
m_{1} \omega^{2}-2 K & 2 K \cos \left(k_{x} a / 2\right) & -\frac{4}{\pi} i K R \sin \left(k_{x} a / 2\right) \\
2 K \cos \left(k_{x} a / 2\right) & m_{2} \omega^{2}-2 K & 0 \\
\frac{4}{\pi} i K R \sin \left(k_{x} a / 2\right) & 0 & I_{2} \omega^{2}-2 K R^{2}
\end{array}\right| \\
& =0 \text {, }
\end{aligned}
$$

where $k_{x}$ is the Bloch wave vector along the $\Gamma X$ direction. By solving Eq. (2) we establish the dispersion relations, that is, $\omega(k)$, for which the expression is complicated in general except for those at the high symmetry points. For example, at

the $\Gamma$ point $\left(k_{x}=0\right)$, Eq. (2) gives three eigenfrequencies in terms of the material parameters: $\omega_{1 \Gamma}=0, \omega_{2 \Gamma}=\sqrt{2 K R^{2} / I_{2}}$, $\omega_{3 \Gamma}=\sqrt{2 K\left(m_{1}+m_{2}\right) /\left(m_{1} m_{2}\right)}$. The corresponding results at the $X$ point of the reduced Brillouin zone $\left(k_{x}=\pi / a\right)$ are $\omega_{1 X}=\sqrt{2 K / m_{2}}, \omega_{2 X}=\sqrt{2 K\left(R^{2} / I_{2}-\Delta\right)}$, and $\omega_{3 X}=$ $\sqrt{2 K\left(1 / m_{1}+\Delta\right)}$, where $\Delta$ is a positive quantity and equals $\left[\sqrt{\left(I_{2}-R^{2} m_{1}\right)^{2}+16 m_{1} I_{2} R^{2} / \pi^{2}}-\left(I_{2}-R^{2} m_{1}\right)\right] / 2 m_{1} I_{2}$.

\section{RESULTS AND DISCUSSION}

\section{A. Band structure and eigenstates}

We test the validity of the lumped model by using a common EPC: a square array of steel cylinders with radius $R=0.2 a$ embedded in epoxy. The mass density, longitudinal, and transverse velocities inside the epoxy are, respectively, $\rho_{1}=1180 \mathrm{~kg} / \mathrm{m}^{3}, c_{l 1}=2540 \mathrm{~m} / \mathrm{s}$, and $c_{t 1}=1160 \mathrm{~m} / \mathrm{s}$. The corresponding parameters of the steel are $\rho_{2}=7900 \mathrm{~kg} / \mathrm{m}^{3}$, $c_{l 2}=5800 \mathrm{~m} / \mathrm{s}$, and $c_{t 2}=3200 \mathrm{~m} / \mathrm{s}$. We use COMSOL Multiphysics, a commercial package based on the finite-element method, to compute the band structure of this EPC and plot the results in circles in Fig. 2(a). The solid circles highlight the transverse and rotational branches of interest, and the open circles represent the longitudinal modes. The band structure can also be evaluated from the lumped model, that is, the solution of Eq. (2), in which the masses are $m_{1}=\rho_{1} a(a-2 R)$ and $m_{2}=\rho_{2} \pi R^{2}$. Here $m_{1}$ is chosen to be the mass of the portion of the matrix that is sandwiched between two adjacent scatterers, which, as will be shown later, effectively contributes to the transverse motion. In this case, $m_{2}$ is about 1.4 times that of $m_{1}$. Consequently, the previously derived $\omega_{2 \Gamma}$ is smaller than $\omega_{3 \Gamma}$. To solve Eq. (2) we need to know the spring constant $K$, which should be determined from the moduli of the materials, but the relation is complex and beyond the scope of this work. Nevertheless, we shall fit its value by choosing one point from the band structure. Here, for simplicity, we adopt the eigenstate at the $\Gamma$ point on the second branch and let its frequency equal the lumped model prediction $\omega_{2 \Gamma}$. Thus, the value of $K$ can be evaluated from $\omega_{2 \Gamma}=\sqrt{2 K R^{2} / I_{2}}=\sqrt{4 K / m_{2}}$. With $m_{1}$, $m_{2}$, and $K$, it is straightforward to compute the dispersion relations from the lumped model. The results are plotted in Fig. 2(a) in solid red curves, which agree well with the solid circles, suggesting the lumped model is valid for the lowest three transverse and rotational branches near the $\Gamma$ point.

To gain a deeper understanding of the highlighted branches, we plot in Figs. 2(b)-2(g) the displacement field patterns associated with six eigenstates at high symmetry points of the Brillouin zone. Color represents the normalized magnitude, with dark red and dark blue corresponding to the maximium (normalized to 1) and zero, respectively, and the small arrows indicate the direction of the displacements. The motions of the cylinder and the matrix can be qualitatively described by the thick arrows. The upper panel corresponds to states at the $\Gamma$ point and the lower panel shows those at the $X$ point. From left to right, the corresponding frequency is increasing. In Fig. 2(a) we mark these numerically simulated eigenstates in a fashion of "symbol followed by a number" to distinguish from those subscripts used in identifying the eigenfrequencies derived from the lumped model. Figures 2(b) and 2(c) show translational movement along the direction perpendicular to 


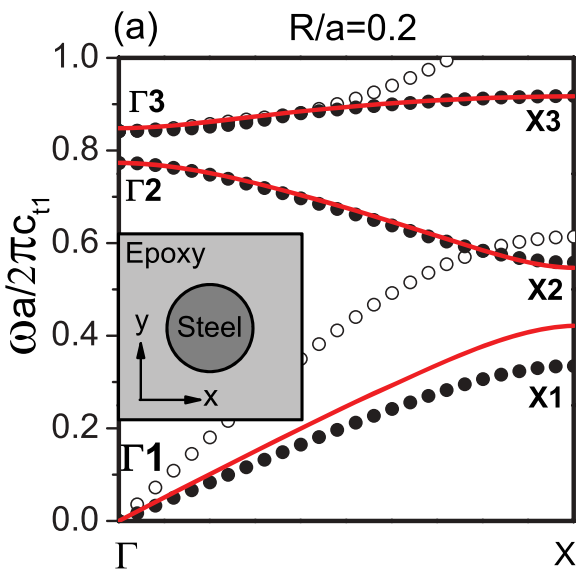

(b)

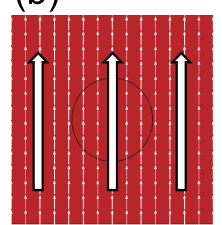

(c)

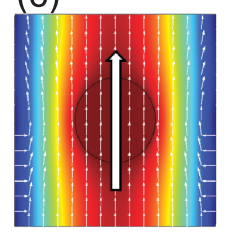

(d)

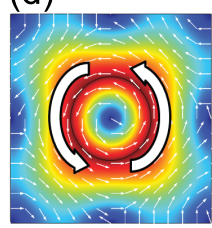

(e)

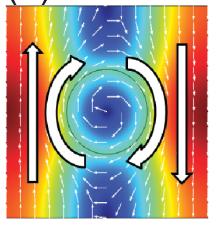

(f)

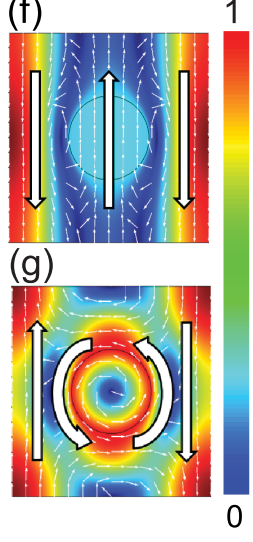

FIG. 2. (Color online) (a) Band structures of a square array of steel cylinders embedded in epoxy with radius $R / a=0.2$. The solid dots correspond to the transverse- and rotation-related states. The red curves indicate the dispersion relations predicted by the lumped model. (b)-(g) The displacement field distributions of the eigenstates marked in (a). The correspondences are: (b) $\Gamma 1$, (c) $X 1$, (d) $\Gamma 2$, (e) $X 2$, (f) $\Gamma 3$, (g) $X 3$. Dark red and dark blue corresponds to one and zero of the normalized magnitude, respectively, and thin arrows indicate directions. Thick arrows demonstrate, qualitatively, the movement of the matrix and the scatterer.

the wave vector $\mathbf{k}$. Near the $\Gamma$ point, the scatterer and the matrix move in unison, and at the Brillouin zone boundary the movement is concentrated in the scatterer. These states are close to the typical states on the acoustic branch of a diatomic chain. ${ }^{26}$ After examining several other states on the branch calculated by either method, we found it is indeed an acoustic-type branch coupled with some rotations. Different from Figs. 2(b) and 2(c), pure rotation of the scatterer is found in Fig. 2(d), which is in accordance with the lumped model as frequency $\omega_{2}$ is the natural angular frequency of an oscillating rotating cylinder and is a function of the moment of inertia. The state on the same branch but at the Brillouin zone boundary is exhibited in Fig. 2(e), where the rotational motion of the scatterer is preserved but is weak compared with the strong translational displacements of the matrix. The matrices on the left and right sides are moving in the opposite directions as if they drive the rotational motion of the scatterer. We call this type of state "in-phase," which is a contrast to the state plotted in Fig. 2(g), where the rotation of the scatterer is not in line with the translational displacement of the matrix and we call it the "out-of-phase" mode. Figure 2(f) demonstrates a state that has a similar pattern of the "optical-type" mode, ${ }^{26}$ where the scatterer and the matrix are moving both vertically but in opposite directions. This again agrees with the lumped model prediction, which gives the typical frequency, that is, $\omega_{3 \Gamma}$, of an optical mode of a diatomic chain. ${ }^{26}$ In fact, this state is the transverse state of the two degenerated dipolar modes. The other one depicted in Fig. 2(a) by the open circles near state $\Gamma 3$ is longitudinal with the matrix and the scatterer moving horizontally, which is not considered here.

Although Fig. 2(a) displays surprisingly good agreement between the numerical simulation and the model, we would like to point out that it does not imply the model is accurate everywhere for an arbitrary case. It is worth mentioning the limitation of the current model. In this lumped model, two types of material parameters, mass and spring constant, play important roles in computing the dispersion relations. In the above case we choose relatively easy ways to determine their values at the expense of losing some accuracy. For example, Figs. 2(e) and 2(f) indicate that the matrix above or below the scatterer contributes very little to the transverse movement so that the choice of $m_{1}$ is justified, but this is not true for the states shown in Figs. 2(b) and 2(c), in which a quite large amount of the matrix that participates in the vibration together with $m_{2}$ was not considered or even was wrongly included as a portion of $m_{1}$. This example indicates the selection of the masses may not be universal or exact. The situation is similar for the determination of the spring constant $K$. The real case is far more complex than our assumption of constant $K$, which we believe to be frequency dependent. For instance, for the case we mentioned earlier, if we choose $\Gamma 1$ and $\Gamma 3$ as the fitting points to fit for a $K$, we would get $K_{\Gamma 1}: K_{\Gamma 2}: K_{\Gamma 3}=0.82: 1: 0.99 .{ }^{27}$ This implies $K$ is frequency dependent, and it does not vary too much in this case so that we were able to get a relatively good agreement between the model with a constant $K$ and the numerical simulations as shown in Fig. 2(a). In a general case, to get exact dispersion relations from the lumped model, one needs to know the accurate information on the masses and the spring constant, both of which are complicated functions of frequency. Therefore, using a constant lumped model, though convenient, is somewhat limited in describing such a continuous system. In principle, this limitation can be reduced by adopting different parameters at different frequencies. In practice, however, it is not easy to do so for some states, such as the one displayed in Fig. $2(\mathrm{~g})$, in which accurate $m_{1}$ is hard to get. Among the states at high symmetry points, those at $X$ points involve higher uncertainty in choosing the parameters than those at the $\Gamma$ point. This would result in some inaccuracy of the model near the $X$ point. Nevertheless, the model can still qualitatively describe the vibration behaviors at the $X$ point. Fortunately, the states at the $\Gamma$ point exhibit pure translational or rotational movements that enable us to pick up proper parameters for the lumped model much more easily. Thus, with the proper parameters chosen from the states at the $\Gamma$ point, the model is able to accurately predict the band structures near the $\Gamma$ point. 


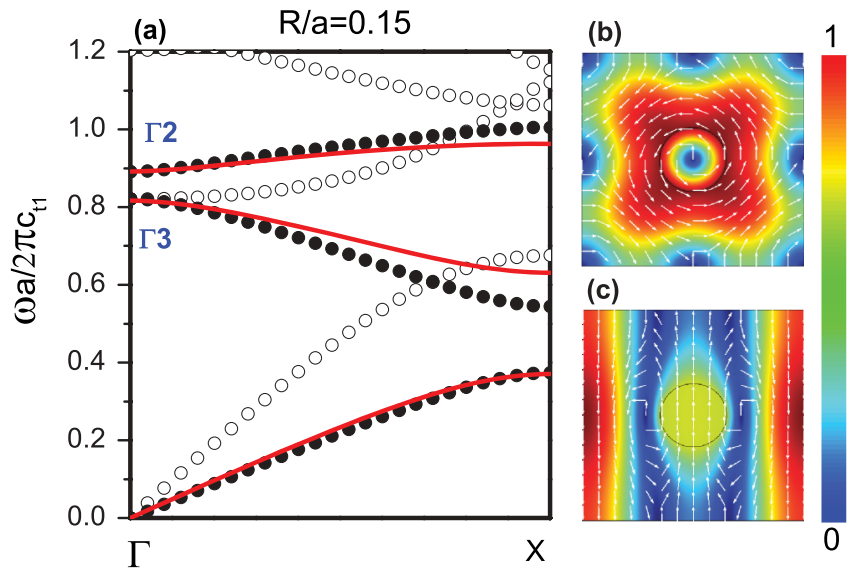

FIG. 3. (Color online) (a) Band structures of the same system as shown Fig. 2, but the radius is changed to $R / a=0.15$. (b) and (c) The displacement field distributions of the eigenstates marked as $\Gamma 2$ and $\Gamma 3$, respectively.

Figure 2(a) shows a band gap between the second and the third band. The lumped model suggests that the width of the gap is determined by the difference between the two masses, that is, $\Delta \omega=\omega_{3 \Gamma}-\omega_{2 \Gamma}=\sqrt{2 K}\left(\sqrt{1 / m_{1}}-\sqrt{1 / m_{2}}\right)$. For the EPC we have just studied, $m_{1}$ is smaller than $m_{2}$. The rotational mode is therefore located below the optical-type mode at the $\Gamma$ point. If $m_{1}$ is greater than $m_{2}$, these modes will interchange their positions. Given the materials, the values of $m_{1}$ and $m_{2}$ are simply functions of the size of the scatterer. Figure 3(a) shows the band structure of a similar EPC but the radius of the steel cylinder is changed to $0.15 a$. Here, for simplicity, we adopted the same method we used for the previous example to obtain the dispersion relations from the lumped model. Good agreement between the lumped model and the numerical calculation is seen again, except for the region near the $X$ point, which is due to the limitation of the model. In this case, $m_{1} / m_{2} \approx 1.45$ and $\omega_{2 \Gamma}>\omega_{3 \Gamma}$. Figures $3(\mathrm{~b})$ and 3(c) show the field patterns of the states marked as $\Gamma 2$ and $\Gamma 3$, respectively. Obviously, the one at the lower frequency is an "optical-type" transverse mode degenerating with a longitudinal mode and the other is a rotational mode. This is opposite to the case shown in Fig. 2.

\section{B. Zero gap: A Dirac-like cone}

Since the magnitudes of $\omega_{2 \Gamma}$ and $\omega_{3 \Gamma}$ depend on the scatterer's size, it is possible to make them equal by carefully tuning the radius of the cylinder. Thus, an interesting result, the zero gap width, is achievable as shown in Fig. 4(a), where the radii of the steel rods are $0.177 a$, very close to the lumped model prediction of $0.176 a$. As expected, we find the rotational mode and the dipolar modes occur simultaneously at a dimensionless frequency $\omega_{0} a / 2 \pi c_{t 1}=0.826$ at the $\Gamma$ point. A bit surprising result is that in the vicinity of this frequency, the dispersion relations become linear as plotted in an enlarged view in Fig. 4(b). An equifrequency surface at a frequency slightly below $\omega_{0}$, that is, $\omega a / 2 \pi c_{t 1}=0.82$, is plotted in Fig. 4(c), with the blue circles indicating the results calculated by COMSOL. These circles lie on a perfect and large circle drawn in a red solid curve, which implies that the dispersion relation is isotropic. Similar results are also obtained for the upper branch but are not shown here. The isotropic behavior of the equifrequency surface somehow contradicts our common understanding that the dispersion relation of an EPC in a square lattice is in general anisotropic. ${ }^{15,16,28}$ In fact, this special characteristic is a result of accidental degeneracy and ensures that the dispersion relation near the degenerate frequency can be described in terms of a linear cone that intersects a flat sheet in the vicinity of the zone center, which is also called a "Diraclike" cone and the vertex of the cone is called a Dirac-like point. $^{21}$ This behavior looks similar to the Dirac-like cone of electromagnetic ${ }^{20}$ and acoustic waves, ${ }^{6}$ where the monopolar and dipolar bands meet at the $\Gamma$ point. Dirac-like cones were also found in an EPC when the accidental degeneracy of the
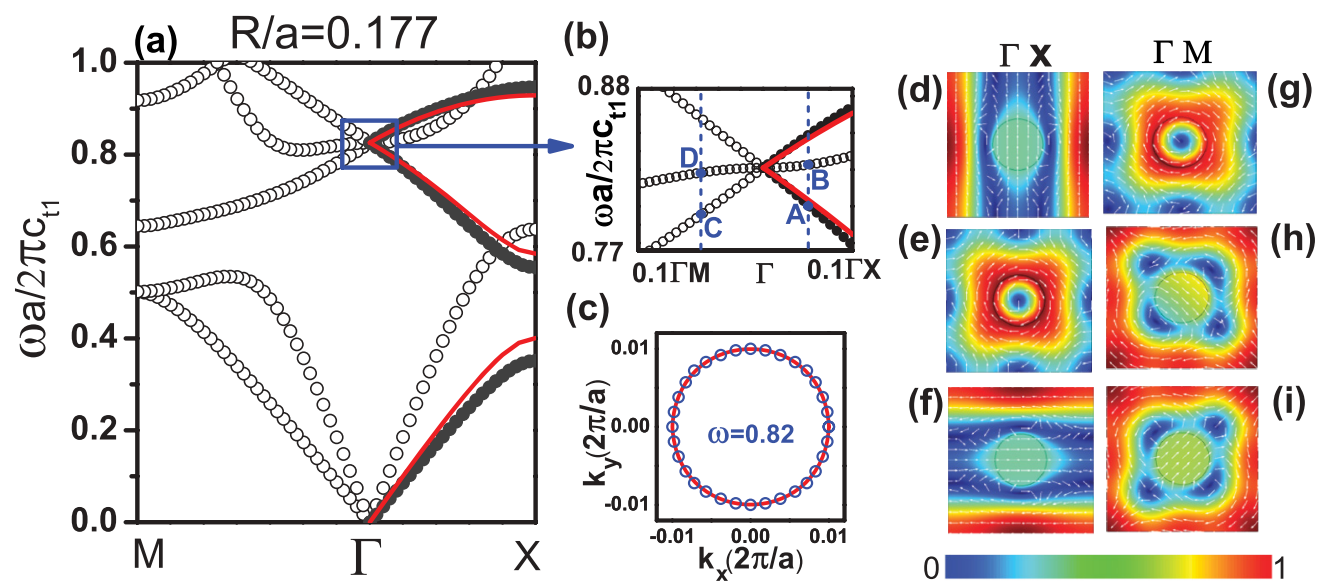

FIG. 4. (Color online) (a) Full band structures of the same system as shown in Fig. 2, but the radius is changed to $R / a=0.177$. (b) An enlarged view of the band dispersion near the Dirac-like point. (c) The equifrequency surface at the dimensionless frequency $\omega a / 2 \pi c_{t 1}=0.82$. The blue circles indicate the numerical calculations, which form a perfect circle plotted in red curve. (d)-(f) The displacement field distribution of the eigenstates near the Dirac point along the $\Gamma X$ direction. (d) and (e) The real part and the imaginary parts of displacement fields of state A. (f) The real part of the displacement field of state B. (g)-(i) Corresponding results for states along the $\Gamma M$ direction. (g) and (h) The real part and the imaginary parts of the displacement field of state C. (i) The real part of the displacement field of state D. 
dipolar and quadrupolar modes occurs and was understood from an effective medium perspective. ${ }^{14}$ In that case, the effective mass density $\rho_{\text {eff }}$ and the effective stiffness $1 / C_{44}$ are simultaneously zero at the Dirac-like point. Here we discover Dirac-like cones for elastic waves, which is a result of the accidental degeneracy of rotational and dipolar modes. The occurrence of the Dirac-like point cannot be interpreted by conventional effective medium theory, ${ }^{11,14}$ however, because the wavelength in the steel rods is longer than that in the epoxy matrix, deeming the conventional effective medium description inapplicable. It is, on the other hand, well predicted by the lumped model and the dispersion relation can also be evaluated by the lumped model as shown by the solid red lines in Figs. 4(a) and 4(b).

Figures 4(d)-4(i) show the displacement field patterns near the Dirac-like point with a small $\mathbf{k}$ along $\Gamma X$ direction (left panel) and $\Gamma M$ direction (right panel). Figures 4(d) and 4(e) show the real and imaginary parts of the displacement fields of the state on the lower branch [marked as A in Fig. 4(b), where $k_{x}=0.05 \pi / a$, and $\left.k_{y}=0\right]$. It is clear that this eigenstate is a linear combination of dipole and rotation excitation. The real part of the displacement is concentrated within the matrix along the direction perpendicular to $\mathbf{k}$. Thus, it can be regarded as a transverse component, which is similar to the transverse dipolar mode mentioned previously [as shown in Fig. 2(f)]. Similarly, the imaginary part can be viewed as a rotational component, in which the motion is mainly the rotation of the steel rod. Because the eigenstates with the same $\mathbf{k}$ but on the upper branch exhibit the same behavior, we do not plot them here. Figure 4(f) shows the real part of the displacement of the state on the middle branch [marked as B in Fig. 4(b)]. Interestingly, a longitudinal dipolar mode with its vibration direction parallel to $\mathbf{k}$ is found. The imaginary part of state $\mathrm{B}$ is two orders of magnitude smaller than its real part and is not presented. The eigenstate along the $\Gamma M$ direction is plotted in Figs. 4(g) and 4(h) for the real and imaginary parts of the state that belong to the lower branch of the linear cone [marked as C in Fig. 4(b), whose $k_{x}=0.05 \pi / a$ and $k_{y}=$ $0.05 \pi / a]$. The behavior of the displacement fields is in general similar to the one along the $\Gamma X$ direction, but the rotation and transverse component interchange their positions, that is, the real part represents a rotation mode, whereas the imaginary part represents a transverse mode. Similar eigenstates are found on the upper branch with the same k. In the middle branch, the real part of the state marked as D in Fig. 4(b) is plotted in Fig. 4(i), whose vibration direction is parallel to k. The imaginary part of this state is, again, very small compared with the real part. Together these observations suggest the remarkable properties of this Dirac-like cone: The linear cone is mainly contributed by the hybrid states of the rotational and transverse components, and the middle branch is longitudinal. This means that the isotropic linear cone can only be coupled with the transverse wave in all directions.

\section{CONCLUSIONS AND OUTLOOK}

In this work we propose a simple lumped model that can account for the local rotation of the scatterer in certain two-dimensional EPCs. The model is useful for a physical understanding of the lowest transverse and rotational bands. It also provides a good estimation of the dispersion relations. With the model, it is convenient to go beyond the translational degree of freedom and utilize the rotational mode to achieve interesting wave propagation properties. As an example, we demonstrate the occurrence of a new type of Dirac-like cone, which is a result of the accidental degeneracy of the rotational and dipolar modes at the Brillouin zone center. This Dirac-like cone possesses isotropic dispersion relations that can be excited only by transverse waves in the vicinity of the Dirac-like point even for a square lattice, which might lead to interesting elastic wave transport properties.

\section{ACKNOWLEDGMENTS}

The authors would like to thank Professor P. Sheng, Professor Z. Q. Zhang, Professor Z. Y. Liu, and Dr. C. Y. Qiu for discussions. The work described here was supported by the KAUST Baseline Research Fund, National Natural Science Foundation of China (Grants No. 10804086 and No. 11274120), and the Fundamental Research Funds for the Central Universities (Grant No. 2012ZZ0077).

\footnotetext{
*ying.wu@kaust.edu.sa

${ }^{1}$ J. V. Sánchez-Pérez, D. Caballero, R. Martinez-Sala, C. Rubio, J. Sanchez-Dehesa, F. Meseguer, J. Llinares, and F. Galvez, Phys. Rev. Lett. 80, 5325 (1998); F. Cervera, L. Sanchis, J. V. Sanchez-Perez, R. Martinez-Sala, C. Rubio, F. Meseguer, C. Lopez, D. Caballero, and J. Sanchez-Dehesa, ibid. 88, 023902 (2001); D. Torrent, A. Hakansson, F. Cervera, and J. Sanchez-Dehesa, ibid. 96, 204302 (2006).

${ }^{2}$ S. Yang, J. H. Page, Z. Liu, M. L. Cowan, C. T. Chan, and P. Sheng, Phys. Rev. Lett. 93, 024301 (2004); J. Mei, Z. Liu, W. Wen, and P. Sheng, ibid. 96, 024301 (2006).

${ }^{3}$ X. Hu, C. T. Chan, and J. Zi, Phys. Rev. E 71, 055601(R) (2005).

${ }^{4}$ M. Kafesaki and E. N. Economou, Phys. Rev. B 60, 11993 (1999).

${ }^{5}$ J. Li and C. T. Chan, Phys. Rev. E 70, 055602(R) (2004).
}

${ }^{6}$ F. M. Liu, X. Q. Huang, and C. T. Chan, Appl. Phys. Lett. 100, 071911 (2012).

${ }^{7}$ Y. Wu, J. Mei, and P. Sheng, Physica B: Condens. Matter 407, 4093 (2012). J. Mei, Y. Wu, and Z. Liu, Europhys. Lett. 98, 54001 (2012).

${ }^{8}$ L. Feng, X.-P. Liu, M.-H. Lu, Y.-B. Chen, Y.-F. Chen, Y.-W. Mao, J. Zi, Y.-Y. Zhu, S.-N. Zhu, and N.-B. Ming, Phys. Rev. Lett. 96, 014301 (2006); M.-H. Lu, C. Zhang, L. Feng, J. Zhao, Y.-F. Chen, Y.-W. Mao, J. Zi, Y.-Y. Zhu, S.-N. Zhu, and N.-B. Ming, Nat. Mater. 6, 744 (2007).

${ }^{9}$ M. S. Kushwaha, P. Halevi, L. Dobrzynski, and B. Djafari-Rouhani, Phys. Rev. Lett. 71, 2022 (1993); M. S. Kushwaha, P. Halevi, G. Martínez, L. Dobrzynski, and B. Djafari-Rouhani, Phys. Rev. B 49, 2313 (1994).

${ }^{10}$ Z. Liu, X. Zhang, Y. Mao, Y. Zhu, Z. Yang, C. T. Chan, and P. Sheng, Science 289, 1734 (2000); J. Mei, Z. Liu, J. Shi, and 
D. Tian, Phys. Rev. B 67, 245107 (2003); Z. Liu, C. T. Chan, and P. Sheng, ibid. 71, 014103 (2005).

${ }^{11}$ Y. Wu, Y. Lai, and Z. Q. Zhang, Phys. Rev. B 76, 205313 (2007); Phys. Rev. Lett. 107, 105506 (2011).

${ }^{12}$ G. Wang, X. Wen, J. Wen, L. Shao, and Y. Liu, Phys. Rev. Lett. 93, 154302 (2004); H. Zhao, Y. Liu, G. Wang, J. Wen, D. Yu, X. Han, and X. Wen, Phys. Rev. B 72, 012301 (2005).

${ }^{13}$ C. Croënne, E. D. Manga, B. Morvan, A. Tinel, B. Dubus, J. Vasseur, and A.-C. Hladky-Hennion, Phys. Rev. B 83, 054301 (2011).

${ }^{14}$ F. Liu, Y. Lai, X. Huang, and C. T. Chan, Phys. Rev. B 84, 224113 (2011).

${ }^{15}$ Y. Wu and Z. Q. Zhang, Phys. Rev. B 79, 195111 (2009).

${ }^{16}$ Q. Ni and J. C. Cheng, Phys. Rev. B 72, 014305 (2005).

${ }^{17}$ Y. Lai, Y. Wu, P. Sheng, and Z. Q. Zhang, Nat. Mater. 10, 620 (2011).

${ }^{18}$ X. Liu, G. Hu, G. Huang, and C. T. Sun, Appl. Phys. Lett. 98, 251907 (2011).

${ }^{19}$ A. L. Fetter and J. D. Walecka, Theoretical Mechanics of Particles and Continua (Dover, New York, 2003).
${ }^{20}$ X. Huang, Y. Lai, Z. Hang, H. H. Zheng, and C. T. Chan, Nat. Mater. 10, 582 (2011).

${ }^{21}$ J. Mei, Y. Wu, C. T. Chan, and Z. Q. Zhang, Phys. Rev. B 86, 035141 (2012).

${ }^{22}$ G. W. Milton and J. R. Willis, Proc. R. Soc. A 463, 855 (2007).

${ }^{23}$ H. H. Huang and C. T. Sun, Philos. Mag. 91, 981 (2011).

${ }^{24}$ L. M. Schwartz, D. L. Johnson, and S. Feng, Phys. Rev. Lett. 52, 831 (1984).

${ }^{25}$ A. Merkel, V. Tournat, and V. Gusev, Phys. Rev. E 82, 031305 (2010); V. Tournat, I. Pèrez-Arjona, A. Merkel, V. SanchezMorcillo, and V. Gusev, New J. Phys. 13, 073042 (2011); A. Merkel, V. Tournat, and V. Gusev, Phys. Rev. Lett. 107, 225502 (2011).

${ }^{26} \mathrm{C}$. Kittel, Introduction to Solid State Physics, 8th ed. (John Wiley, New York, 2005)

${ }^{27}$ Since $\omega_{1 \Gamma}=0$ we take the zero frequency limit of the lumped model and use the slope of the band structure to fit for a $K$.

${ }^{28}$ L. D. Landau and E. M. Lifshitz, Theory of Elasticity (ButterworthHeinemann, Oxford, 1986). 